\title{
Spectral simulations of polar diatomic molecules immersed in He clusters: application to the $\mathrm{ICl}(X)$ molecule
}

\author{
P Villarreal ${ }^{1}$, M P de Lara-Castells ${ }^{1}$, R Prosmiti $^{1}$, G Delgado-Barrio ${ }^{1}$, \\ D López-Durán ${ }^{1}$, F A Gianturco ${ }^{2}$ and J Jellinek ${ }^{3}$ \\ ${ }^{1}$ Instituto de Matemáticas y Fásica Fundamental (CSIC), Serrano 123, E-28006-Madrid, Spain \\ ${ }^{2}$ Department of Chemistry and INFM, The University of Rome, Città Universitaria, 00185, Rome, Italy \\ ${ }^{3}$ Chemistry Division, Argonne National Laboratory, Argonne, IL 60439, USA \\ E-mail: p.villarreal@imaff.cfmac.csic.es
}

Received 28 March 2007

Accepted for publication 18 April 2007

Published 13 August 2007

Online at stacks.iop.org/PhysScr/76/C96

\begin{abstract}
A recently developed quantum-chemistry-like methodology to study molecules solvated in atomic clusters is applied to the $\mathrm{ICl}$ (iodine chloride) polar diatomic molecule immersed in clusters of He atoms. The atoms of the solvent clusters are treated as the 'electrons' and the solvated molecule as a structured 'nucleus' of the combined solvent-solute system. The helium-helium and helium-dopant interactions are represented by parametrized two-body and $a b$ initio three-body potentials, respectively. The ground-state wavefunctions are used to compute the infrared (IR) spectra of the solvated molecule. In agreement with the experimental observations, the computed spectra exhibit considerable differences depending on whether the solvent cluster is comprised of bosonic $\left({ }^{4} \mathrm{He}\right)$ or fermionic $\left({ }^{3} \mathrm{He}\right)$ atoms. The source of these differences is attributed to the different spin-statistics of the solvent clusters. The bosonic versus fermionic nature of the solvent is reflected in the IR absorption selection rules. Only $\mathrm{P}$ and $\mathrm{R}$ branches with single state transitions appear in the spectrum when the molecule is solvated in a bosonic cluster. On the other hand, when the solvent represents a fermionic environment, quasi-degenerate multiplets of spin states contribute to each branch and, in addition, the Q-branch becomes also allowed. Combined, these two factors explain the more congested nature of the spectrum in the fermionic case.
\end{abstract}

PACS numbers: 36.40.Mr, 31.15.Ar, 33.20.Fb, 33.80.Gj

\section{Introduction}

The spectroscopic study of molecules in ultracold environments constitute a field of current increasing interest. After the pioneering works on infrared (IR) spectra of $\mathrm{SF}_{6}$ molecules immersed in helium nanodroplets [1,2], further advances in the synthesis and characterization of solvated molecular species have stimulated the activity in this field $[3,4]$. Rotationally resolved IR spectra of different molecules in helium nanodroplets [5-7], and high-resolution IR measurements on $\mathrm{CO}$ in small He clusters $[8,9]$ have been recently reported. In particular the IR spectra of OCS $[10,11]$ reveal interesting features that relate to the quantum nature of the solvent environment. So, when OCS is solvated in a fermionic ${ }^{3} \mathrm{He}$-nanodroplet, the spectrum shows an unstructured broad shape which resembles those of heavy molecules immersed in liquids. On the contrary, the spectrum of OCS in a bosonic ${ }^{4} \mathrm{He}$-nanodroplet is very similar to that of the gas phase OCS and displays well-defined P and R branches. It looks as if the molecule executes an essentially free rotation in the bosonic solvent which has been interpreted as an indication of superfluidity of the ${ }^{4} \mathrm{He}$-nanodroplet $[10,11]$.

From the theoretical side, the structure and energetics of both pure and doped ${ }^{4} \mathrm{He}$ clusters is currently described through a variety of robust quantum mechanical approaches. These include diffusion Monte Carlo (DMC) techniques [12], finite temperature Feynman path integral based Monte Carlo methodologies [13], or density functional theory approaches (a recent review can be found at [14]). These different frameworks, however, were developed for and applied to 
primarily, if not exclusively, bosonic ${ }^{4} \mathrm{He}$ clusters. The reason for this is the added complexity of the fermionic ${ }^{3} \mathrm{He}$ systems. In the case of DMC, for example, this complexity exhibits itself in the nodal structure of the wavefunction. A simplifying partial remedy is to use the fixed-node approximation [15] that furnishes an upper bound to the ground-state energy. The quality of the results obtained within this approximation depends on the choice of the trial wavefunction; the effect of the approximation can be evaluated by the released-node technique [16].

A very different, alternative approach is based on viewing and treating the cluster-dopant system as a 'molecular species', in which the atoms of the solvent cluster play the role of the electrons and the atoms of the dopant molecule serve as the nuclei. The difference with a true molecule is that one has to replace the electrostatic interactions by the corresponding interatomic or molecular interactions. Such an alternative, quantum chemistry-like technique was first formulated and applied to characterize the lowest energy triplet state of the $\mathrm{SF}_{6}-\left({ }^{3} \mathrm{He}\right)_{2}$ system [17]. A similar technique, but based on the configuration interaction methodology, was used to study anthracene- $\left({ }^{\epsilon} \mathrm{He}\right)_{2}, \epsilon=4,3$, complexes $[18,19]$. It was also applied in a recent variational study of rigid $\mathrm{Br}_{2}$ molecules surrounded by up to five ${ }^{4} \mathrm{He}$ atoms [20].

By implementing Hartree-Fock and Hartree methodology for fermionic and bosonic environments, respectively, we have used this idea to study much larger He clusters doped with $\mathrm{BC}$ diatomic molecules, where $\mathrm{B}$ and $\mathrm{C}$ are hologen atoms, as $\mathrm{Br}_{2}$ [21-23] or $\mathrm{ICl}[24,25]$. In this way, the quantal nature of the surrounding $\mathrm{He}$ atoms is accounted for on the same footing as in the electronic problem. Another merit of this new scheme is that, since it furnishes the wavefunction of the combined system, it allows not only for characterization of the structural and energy properties, but also for performing spectra simulations, which can be directly compared with measured data. By simulating IR absorption cross-section for polar molecules as a function of the incident photon energy [24], a clear distinction between bosonic or fermionic environments can be anticipated through the corresponding selection rules. In the former scenario, the allowed transitions are those corresponding to the isolated molecule, though the spectral lines of the solvated molecule get shifted and broadened as a consequence of the interaction of the molecule with the solvent. In a fermionic environment, additional new transitions become allowed and therefore contribute to the congestion of the final spectrum.

Hartree calculations and IR spectral simulations for ${ }^{4} \mathrm{He}_{N}-\mathrm{ICl}$ bosonic clusters, $N \leqslant 30$, have been recently reported [24]. Concerning the potential energy surface (PES), ab initio computations on the $\mathrm{He}_{2}-\mathrm{ICl}$ tetra-atomic cluster [26] show that the interaction energy can be accurately described as a sum of the $\mathrm{He}-\mathrm{ICl}$ triatomic and $\mathrm{He}-\mathrm{He}$ pair interactions. Hence, for clusters containing several He atoms, the full PES is constructed as the sum of the $\mathrm{ICl}$ intramolecular potential, all the triatomic $\mathrm{He}-\mathrm{ICl}$ intermolecular potentials, and all the $\mathrm{He}-\mathrm{He}$ interactions. For the $\mathrm{He}-\mathrm{ICl}(X)$ triatomic cluster, $a b$ initio calculations [27] predict three PES minima, which correspond to 'linear' (He on the side of the I end of the ICl molecule), near T-shaped, and 'anti-linear' (He on the side of the $\mathrm{Cl}$ end of the $\mathrm{ICl}$ molecule) equilibrium structures.
We found that this high degree of anisotropy has a persistent effect on the clusters for all the sizes (values of $N$ ) considered.

In this paper an overview of this approach is presented and extended to the case of ${ }^{3} \mathrm{He}_{N}-\mathrm{ICl}$ fermionic clusters. The conceptual framework and theoretical models, including the methodology to perform the spectra simulations, are outlined in section 2. To be close to the experiments an extension to much bigger clusters is conjectured, and the simulated spectra at extremely low temperatures depending on the quantum nature of the environment are presented and discussed in section 3. Finally, concluding remarks and an outlook of future work are given in section 4 .

\section{Theoretical models}

\subsection{Adiabatic quantum-chemistry-like treatment}

Using satellite coordinates $\left\{\left(\mathbf{r}, \mathbf{R}_{k}\right)\right\}$, where $\mathbf{r}$ is the vector joining the $\mathrm{B}$ and $\mathrm{C}$ atoms and $\mathbf{R}_{k}$ are the vectors from the centre of mass of the $\mathrm{BC}$ molecule to the different $\mathrm{He}$ atoms, one can write the Hamiltonian of the $\mathrm{He}_{N}-\mathrm{BC}$ system as

$$
H=-\frac{\hbar^{2}}{2 m} \frac{\partial^{2}}{\partial r^{2}}+U(r)+\frac{\mathbf{j}^{2}}{2 m r^{2}}+\sum_{k=1}^{N} H_{k}^{(\epsilon)}+\sum_{k<l} \tilde{V}_{k l},
$$

consisting of a diatomic part which corresponds to the BC molecule (the first three terms) plus $N^{\epsilon} \mathrm{He}-\mathrm{BC}$ triatomic Hamiltonians, $H_{k}^{(\epsilon)}(k=1, N)$, and the He-He interactions $\tilde{V}_{k l}$ which include a potential term $V_{k l}$ and also a kinetic energy coupling arising from the use of non-Jacobi coordinates [28]. In (1), $m$ is the reduced mass of the diatomic molecule, $\mathbf{j}$ is the angular momentum associated with $\mathbf{r}$, and $U$ represents the intramolecular diatomic potential. The triatomic Hamiltonians have the form

$$
H_{k}^{(\epsilon)}=-\frac{\hbar^{2}}{2 \mu_{\epsilon}} \frac{\partial^{2}}{\partial R_{k}^{2}}+\frac{\mathbf{l}_{k}^{2}}{2 \mu R_{k}^{2}}+W\left(R_{k}, r, \theta_{k}\right),
$$

where $\mu_{\epsilon}$ is the reduced mass of the ${ }^{\epsilon} \mathrm{He}-\mathrm{BC}$ system, $\mathbf{l}_{k}$ is the angular momentum associated with $\mathbf{R}_{k}$, and $W$ represents the atom-diatom intermolecular potential that depends on the pair of $\left(R_{k}, r\right)$ distances and the angle $\theta_{k}$ between the $\mathbf{R}_{k}$ and $\mathbf{r}$ vectors. We choose a body-fixed (BF) coordinate system with the $Z^{B F}$ - axis parallel to $\mathbf{r}$, and denote by $\Lambda$ and $\Sigma$ the quantum numbers associated with the projection of $\mathbf{L}=\sum_{k=1}^{N} \mathbf{l}_{k}$ and the total spin $\mathbf{S}$ on $Z^{B F}$, respectively. The total wavefunction is written as a product

$$
\Psi_{\Lambda S \Sigma v}^{J M}\left(\left\{\mathbf{R}_{k}\right\},\left\{\mathbf{s}_{k}\right\}, \mathbf{r}\right)=\mathcal{D}_{M \Omega}^{J^{*}}\left(\varphi_{r}, \theta_{r}, 0\right) \mathcal{F}_{\Lambda S v}^{J \Sigma}\left(\left\{\mathbf{R}_{k}\right\},\left\{\mathbf{s}_{k}\right\}, r\right),
$$

with $\mathbf{s}_{k}(k=1, \ldots, N)$ denoting the spin coordinates of the $N$ solvent atoms. In (3), the $\mathcal{D}_{M \Omega}^{J}$ functions are Wigner rotation matrices that depend on the polar components of $\mathbf{r}$ in the space-fixed (SF) frame, $J$ is the quantum number associated with the total angular momentum $\mathbf{J}=\mathbf{j}+\mathbf{L}+\mathbf{S}$, while $M$ and $\Omega=\Lambda+\Sigma$ are quantum numbers associated with the projections of $\mathbf{J}$ on $Z^{\mathrm{SF}}$ and $Z^{\mathrm{BF}}$, respectively.

The present treatment takes view of the dopant molecule as being perturbed by the presence of the surrounding $\mathrm{He}$ atoms. It starts from an adiabatic approach which considers $\mathrm{BC}$ as having fixed 'nuclei', and the $\mathcal{F}$ function appearing 
in (3) is written as a simple product $\mathcal{F}_{\Lambda S v}^{J \Sigma}\left(\left\{\mathbf{R}_{k}\right\},\left\{s_{k}\right\}, r\right)=$ $\Phi_{\Lambda S}\left(\left\{\mathbf{R}_{k}\right\},\left\{\mathbf{s}_{k}\right\} ; r\right) \chi_{\Lambda S v}^{J \Sigma}(r)$ where the $\Phi$ function satisfies, at fixed $r$ values, the equation:

$$
\left[\sum_{k=1}^{N} H_{k}^{(\epsilon)}\left(\mathbf{R}_{k} ; r\right)+\sum_{k<l}^{N} \tilde{V}_{k l}-E_{\Lambda S}(r)\right] \Phi_{\Lambda S}\left(\left\{\mathbf{R}_{k}\right\},\left\{\mathbf{s}_{k}\right\} ; r\right)=0,
$$

while the $\chi$ functions describe the vibrations of a diatomic molecule distorted by the presence of the $E_{\Lambda S}(r)$ eigenvalues coming from (4),

$$
\left[-\frac{\hbar^{2}}{2 m} \frac{\partial^{2}}{\partial r^{2}}+U(r)+E_{\Lambda S}(r)+\frac{\left\langle\mathbf{j}^{2}\right\rangle}{2 m r^{2}}-\varepsilon_{\Lambda S v}^{J \Sigma}\right] \chi_{\Lambda S v}^{J \Sigma}(r)=0,
$$

where, neglecting Coriolis couplings, the averaged rotational term is approximated by $[21,22,24]$

$$
\begin{aligned}
\left\langle\mathbf{j}^{2}\right\rangle \approx & \left\langle\Phi_{\Lambda S}\left|\mathbf{L}^{2}\right| \Phi_{\Lambda S}\right\rangle+\hbar^{2}[J(J+1)+S(S+1) \\
& \left.-2\left(\Lambda^{2}+\Sigma^{2}+\Lambda \Sigma\right)\right],
\end{aligned}
$$

with the $\mathbf{L}^{2}$ average being computed by using the distribution of $\mathrm{L}$ values in the $\Phi_{\Lambda S}$ state [23]. Recently [29], the assumptions involved in this approach (i.e. decoupling of the orbital angular momentum from the $\mathrm{BC}$ rotation and adiabaticity of the $\mathrm{BC}$ stretching versus the He motions) have been verified as being realistic for the ${ }^{4} \mathrm{He}_{2}-\mathrm{Br}_{2}(X)$ system.

\subsection{Hartree-Fock and Hartree approaches}

To solve (4), and in order to consider on the same footing fermionic and bosonic clusters, we use the Hartree-Fock and the Hartree approaches, respectively, looking for just the ground state of the system under consideration. For doped ${ }^{3} \mathrm{He}$ clusters [21,22] the nuclear wavefunction is written as a Slater determinant, i.e. an antisymmetrized product of one-fermion spin-orbitals,

$$
\begin{aligned}
\Phi_{\Lambda S}= & \frac{1}{\sqrt{N !}} \operatorname{det}\left[\phi_{1}\left(\mathbf{R}_{1}, \mathbf{s}_{1} ; r\right) \cdots \phi_{i}\left(\mathbf{R}_{i}, \mathbf{s}_{i} ; r\right) \cdots\right. \\
& \left.\phi_{N}\left(\mathbf{R}_{N}, \mathbf{s}_{N} ; r\right)\right]
\end{aligned}
$$

where $\mathbf{s}_{i} \quad(i=1, \ldots, N)$ denote the spin coordinates of the $N$ fermions. In turn, each one-fermion wavefunction $\phi_{i}\left(\mathbf{R}_{i}, \mathbf{s}_{i} ; r\right)$ is a product of a spatial one-particle orbital $\psi_{i}\left(\mathbf{R}_{i} ; r\right)$ and a spin function $\sigma\left(\mathbf{s}_{i}\right)=\alpha\left(\mathbf{s}_{i}\right)$ or $\beta\left(\mathbf{s}_{i}\right)$. Depending on the spin-symmetry of the system, the spatial orbitals are found through the restricted closedshell or open-shell Hartree-Fock methods. On the other hand, the nuclear wavefunction of doped ${ }^{4} \mathrm{He}$ clusters is taken as a symmetrized Hartree product of single-particle wavefunctions [23, 24]. For a generic case, when $N_{i}$ bosons occupy the same single-particle orbital labelled as $i$, the $N$-boson total wavefunction is expressed as

$$
\begin{gathered}
\Phi_{\Lambda 0}^{\left(N_{1}, \ldots, N_{M}\right)=} \frac{1}{\sqrt{\mathcal{N}}} \hat{\mathcal{S}}\left(\prod_{i=1}^{N_{1}} \psi_{1}\left(\mathbf{R}_{i} ; r\right) \prod_{j=N_{1}+1}^{N_{1}+N_{2}} \psi_{2}\left(\mathbf{R}_{j} ; r\right) \cdots\right. \\
\left.\qquad \prod_{k=\left(N_{1}+\cdots+N_{M-1}\right)+1}^{N} \psi_{M}\left(\mathbf{R}_{k} ; r\right)\right),
\end{gathered}
$$

where $\sum_{i}^{M} N_{i}=N, \quad M \leqslant N, \hat{\mathcal{S}}$ is the symmetrization operator, and $1 / \sqrt{\mathcal{N}}$ is a normalization factor. In contrast to the case of fermions (Hund's rule), the ground state always corresponds to that in which all the bosons occupy the same one-particle orbital for the bosonic ${ }^{4} \mathrm{He}$ clusters analysed,

$$
\Phi_{\Lambda 0}=\psi_{1}\left(\mathbf{R}_{1} ; r\right) \cdots \psi_{1}\left(\mathbf{R}_{i} ; r\right) \cdots \psi_{1}\left(\mathbf{R}_{N} ; r\right),
$$

which is here reminiscent of the behaviour of a Bose condensate. In turn, the occupied orbital corresponds to a minimum value of the projection of the bosonic angular momentum on to the molecular axis $(\Lambda=0)$.

For bosons and singlet fermions, and starting from $N=2$, the initial orbitals are those corresponding to independent particles. Here on, the chosen initial orbitals were those obtained from the preceding calculation with $N-2$ particles. For $S \neq 0$ fermions, one starts with the optimized orbitals from the $S-1$ calculation for the same size cluster. In either case, spatial one-particle orbitals are expanded in terms of a finite basis set composed of products of radial and angular functions,

$$
\psi_{i}(\mathbf{R} ; r)=\sum_{n l m} c_{n l m}^{i} G_{n}(R ; r) Y_{l m}(\theta, \phi)
$$

where $Y_{l m}$ are spherical harmonics. The radial $G_{n}(R ; r)$ basis functions are obtained as follows: one solves for the ground level the Schrödinger equation corresponding to the triatomic ${ }^{\epsilon} \mathrm{He}-\mathrm{BC}$ subsystem at fixed orientations $\theta_{n}$,

$$
\left[-\frac{\hbar^{2}}{2 \mu_{\epsilon}} \frac{\partial^{2}}{\partial R^{2}}+W\left(R, \theta_{n} ; r\right)-E_{n}(r)\right] g_{n}\left(R, \theta_{n} ; r\right)=0,
$$

repeating the calculations for $n_{\max }$ different equidistant values of $\theta_{n}$ in the range $[0, \pi]$. An orthogonalization Schmidt procedure applied to the $g_{n}$ functions of (11) leads to an orthonormal basis set of $G_{n}$ functions.

We next stress the differences which exist within the application of Hartree/Hartree-Fock procedures to our case vis à vis electronic structure problems. While the electron-electron and electron-nucleus interactions are, respectively, repulsive and attractive, strong repulsion of both helium-helium and helium-dopant interactions occurs at short distances. This causes that, except for the triplet state of the $\left({ }^{3} \mathrm{He}\right)_{2}-\mathrm{BC}$ complex, Hartree-Fock energies of $\left({ }^{3} \mathrm{He}\right)_{N}-\mathrm{BC}$ clusters using the bare $\mathrm{He}-\mathrm{He}$ potential are unphysically too large and positive in many cases [22, 30, 31]. This problem is overcome by truncating the $\mathrm{He}-\mathrm{He}$ potential at short distances $[22,23]$ where the truncated potential now depends on a single parameter which is fitted to reproduce variational calculations on a cluster containing two bosonic ${ }^{4} \mathrm{He}$ atoms, and thereafter used for all cluster sizes including fermions. The strong He-He repulsion at short range further leads to convergence problems when applied via the standard selfconsistent method to iteratively solve the Fock equations. We would like to underline that the use of a direct minimization procedure [32, 33] was essential to force the systems to convergence to the global minima. 


\subsection{IR spectra simulations}

An heteronuclear molecule $\mathrm{BC}$ that has a permanent dipole moment in the ground electronic state can be promoted from an initial vib-rotational state $|i\rangle$ to a final vib-rotational state $|f\rangle$ within the same electronic state by absorption of one photon of frequency $\omega_{i f}$ that matches the energy difference between the initial and final states: this difference belongs to the IR region. When such a molecule is embedded in a bath of He atoms, we can envisage a similar process:

$$
\mathrm{He}_{N}-\mathrm{BC}(i)+\hbar \omega_{i f} \rightarrow \mathrm{He}_{N}-\mathrm{BC}(f),
$$

where now the indices $i$ and $f$ refer, respectively, to the initial and the final states of the entire $\mathrm{He}_{N}-\mathrm{BC}$ cluster. In the electric dipole approximation within the first-order perturbation theory, the absorption intensity is proportional to the square modulus of the matrix element of the transition moment operator, $\boldsymbol{\mu} \cdot \hat{\boldsymbol{e}}$, computed using the $\Psi$ functions defined by (3); here, $\boldsymbol{\mu}$ is the dipole moment of the solvated molecule, and $\hat{e}$ defines the polarization of the electric field. Usually, $\boldsymbol{\mu}$ is expressed in a BF reference frame whereas the electric field defines a natural SF frame. Performing a rotation of the BF frame into the SF frame, $\boldsymbol{\mu}^{\mathrm{SF}}=\boldsymbol{R}^{-1} \boldsymbol{\mu}^{\mathrm{BF}}$, and expressing the $\boldsymbol{\mu}$ and $\hat{\boldsymbol{e}}$ vectors in spherical components, one obtains for the scalar product

$$
\boldsymbol{\mu} \cdot \hat{\boldsymbol{e}}=\sum_{p=-1}^{+1} \sum_{q=-1}^{+1} e_{p}^{*} \mathcal{D}_{p q}^{1 *}\left(\varphi_{r}, \theta_{r}, 0\right) \mu_{q}^{\mathrm{BF}} .
$$

Thus, using $e_{p}^{*}=(-1)^{p} e_{-p}$, the matrix elements of the transition moment become

$$
\begin{gathered}
\left\langle\Psi_{i}|\boldsymbol{\mu} \cdot \hat{\boldsymbol{e}}| \Psi_{f}\right\rangle \propto \sum_{p, q}(-1)^{p} e_{-p}\left\langle\chi_{f}\left|\mu_{q}\right| \chi_{i}\right\rangle \delta_{\Lambda_{f} \Lambda_{i}} \\
\times \int \mathrm{d} \tau \mathcal{D}_{M_{f} \Omega_{f}}^{J_{f}} \mathcal{D}_{p q}^{1 *} \mathcal{D}_{M_{i} \Omega_{i}}^{J_{i} *}
\end{gathered}
$$

As $a b$ initio calculations on $\mathrm{ICl}$ reveal, the dipole moment of the solvated molecule is only weakly affected by the interactions with the surrounding He atoms and its direction remains along the diatomic bond, so that only the $q=0$ component contributes to a parallel transition. If one considers linearly polarized light and uses the direction of polarization to define the SF $Z$-axis ( $p=0)$, one arrives at

$$
\begin{aligned}
& \left\langle\Psi_{i}|\boldsymbol{\mu} \cdot \hat{\boldsymbol{e}}| \Psi_{f}\right\rangle \propto(-1)^{M_{i}}\left\langle\chi_{f}\left|\mu_{0}\right| \chi_{i}\right\rangle \delta_{\Lambda_{f}} \Lambda_{i} \\
& \times\left(\begin{array}{lll}
J_{i} & 1 & J_{f} \\
-M_{i} & 0 & M_{i}
\end{array}\right)\left(\begin{array}{lll}
J_{i} & 1 & J_{f} \\
-\Omega_{i} & 0 & \Omega_{i}
\end{array}\right) .
\end{aligned}
$$

Since $\Omega=\Lambda+\Sigma$, for $\Sigma$ states $(\Lambda=0)$ of bosonic clusters, the second $3-j$ symbol in (13) vanishes unless $\Delta J= \pm 1$. Hence, only the $\mathrm{P}$ and the $\mathrm{R}$ branches survive and the $\mathrm{Q}$ transitions are not allowed in the spectrum within the bosonic scenario. In contrast, when $\Omega \neq 0$ states are involved (e.g. because of the presence of fermionic components in the solvent and/or the presence of $\Pi, \Delta, \ldots$, i.e. $\Lambda \neq 0$ states) the $\mathrm{Q}$ transitions become allowed as well. A Boltzmann distribution of rotational states of the clusters that corresponds to a given temperature $T$ therefore gives rise to a line of intensity

$$
\begin{aligned}
& I_{f i}^{(N)}(T) \propto \frac{\mathrm{e}^{-\left(\varepsilon_{i} / k T\right)}}{\sum_{i} \mathrm{e}^{-\left(\varepsilon_{i} / k T\right)} \frac{1}{2 J_{i}+1}} \\
& \times \sum_{M_{i}}\left|\left\langle\chi_{f}^{(N)}\left|\mu_{0}\right| \chi_{i}^{(N)}\right\rangle\left(\begin{array}{lll}
J_{i} & 1 & J_{f} \\
-M_{i} & 0 & M_{i}
\end{array}\right)\left(\begin{array}{lll}
J_{i} & 1 & J_{f} \\
-\Omega_{i} & 0 & \Omega_{i}
\end{array}\right)\right|^{2}
\end{aligned}
$$

at a frequency $\omega_{f i}=\left(\varepsilon_{\Lambda S v_{f}}^{J_{f} \Sigma}-\varepsilon_{\Lambda S v_{i}}^{J_{i} \Sigma}\right) / \hbar$ (in (14), the simplified notation $\varepsilon_{i}$ stands for $\varepsilon_{\Lambda S v_{i}}^{J_{i} \Sigma}$ ).

Several causes may lead to the broadening of the spectral lines. We assume that the radiation promotes the diatomic subunit to a vibrationally excited state. Its excess of energy can eventually be transferred from the dopant to some of the weak bonds causing their rupture, i.e. the fragmentation of the cluster. This constitutes the mechanism of direct vibrational predissociation (VP). We are aware of the fact that VP competes with other relaxation mechanisms such as, for example, intramolecular energy redistribution, and evaporative cooling [34]. However, to get a rough estimate of the VP width, we extend previous studies on tri-atomic [35] and tetra-atomic [36, 37] species to larger clusters by following the procedure outlined in [21, 22, 24, 25]. First, one calculates the $\theta$-dependent VP width for the ${ }^{\epsilon} \mathrm{He}-\mathrm{BC}\left(X, v_{f}\right)$ triatomic species, $\gamma_{v_{f} \leftarrow v_{i}}$, using the framework of an adiabatic angular model [38]. Then, by integrating over all variables but $\theta$ the square modulus of the function $\Phi_{\Lambda S}\left(\left\{\mathbf{R}_{k}\right\},\left\{\mathbf{s}_{k}\right\} ; r_{\mathrm{eq}}\right)$ one generates an angular distribution $\mathcal{D}_{\Lambda S}^{(N)}(\theta)$, normalized to the number $N$ of solvent atoms, which gives the probability of finding of the solvent around the dopant. The VP line broadening is then obtained by averaging $\gamma_{v_{f} \leftarrow v_{i}}$ over the angular distribution,

$$
\Gamma_{f i}^{(N)}=\int_{0}^{\pi} \mathrm{d} \theta \mathcal{D}_{\Lambda S}^{(N)}(\theta) \gamma_{v_{f} \leftarrow v_{i}}(\theta) .
$$

By dressing the stick lines with Lorentzians of appropriate widths and summing over all transitions, one arrives at a continuum profile for IR photo-absorption cross-section,

$$
\sigma_{N}(\omega ; T)=\frac{1}{2 \pi} \sum_{f, i} \frac{\Gamma_{f i}^{(N)}}{\hbar^{2}\left(\omega-\omega_{f i}\right)^{2}+\left(\Gamma_{f i}^{(N)} / 2\right)^{2}} I_{f i}^{(N)}(T),
$$

which fulfills $\int \mathrm{d} \omega \sigma_{N}(\omega ; T)=\sum_{f, i} I_{f i}^{(N)}(T)$.

\section{Results}

\subsection{PES and numerical details}

As already mentioned, the $\mathrm{He}_{2}-\mathrm{ICl}$ interaction energy [26] is accurately described as the addition of two $\mathrm{He}-\mathrm{ICl}$ potentials [27] plus the $\mathrm{He}-\mathrm{He}$ interaction. This description is extended to clusters of larger sizes and the full PES for a $\mathrm{He}_{N}-\mathrm{ICl}$ cluster is constructed as

$$
\begin{gathered}
V_{\mathrm{He}_{N}-\mathrm{ICl}}\left(\left\{\mathbf{R}_{k}\right\}_{k=1}^{N} ; \mathrm{r}\right)=U(r)+\sum_{k=1}^{N} W_{\mathrm{He}_{\mathrm{k}}-\mathrm{ICl}}\left(\mathbf{R}_{k} ; \mathrm{r}\right) \\
+\sum_{k<l} V_{\mathrm{He}_{\mathrm{k}}-\mathrm{He}_{\mathrm{l}}}\left(\left|\mathbf{R}_{k}-\mathbf{R}_{l}\right|\right) .
\end{gathered}
$$


The $\operatorname{ICl}\left(X^{1} \Sigma^{+}\right)$intramolecular interaction $U(r)$ was approximated by a Morse function [39] with the following values of the parameters: well-depth $D=17557.411 \mathrm{~cm}^{-1}$, characteristic inverse length $\alpha=1.8492349 \AA^{-1}$, and equilibrium distance $r_{e}=2.321 \AA$. In order to define the PES over a range of $r$ values large enough for characterization of the first two ( $v=0$ and 1) vibrational levels of $\operatorname{ICl}(X)$, necessary for the spectral simulations accounted for, a quadratic dependence of the $W$ interaction energy on $r$ has been assumed. In practice, for each orientation $\theta_{i}(i=1-11)$, the coefficients $A$ and $B$ of the expansion

$$
\begin{aligned}
W\left(R, r ; \theta_{i}\right)= & A\left(R, \theta_{i}\right)\left(r-r_{\mathrm{e}}\right)^{2}+B\left(R, \theta_{i}\right)\left(r-r_{\mathrm{e}}\right) \\
& +W\left(R, r_{\mathrm{e}}, \theta_{i}\right)
\end{aligned}
$$

are readily obtained from the corresponding interaction energies $W\left(R, r_{-}, \theta_{i}\right), W\left(R, r_{\mathrm{e}}, \theta_{i}\right)$, and $W\left(R, r_{+}, \theta_{i}\right)$, where $r_{-}$, $r_{\mathrm{e}}$, and $r_{+}$are $2.271,2.321$ and $2.386 \AA$, respectively [24]. Finally for the He-He interaction we employed a parameterized potential of Aziz and Slaman [40]. As mentioned before, the He-He potential was truncated at short distances (for details see [23]).

In the calculations presented here the following masses (amu) were used: $m_{\mathrm{I}}=126.904473, \quad m_{C l}=34.968853$, $m^{{ }^{3} \mathrm{He}}=3.01604$, and $m^{{ }^{4} \mathrm{He}}=4.00260$. A grid of 4096 points in the $R$ range of (2.0-20.0) $\AA$ was employed to numerically solve (11), using a Numerov procedure. We used the following limits in (10) (in parenthesis the corresponding values for fermionic clusters): $\ell_{\max }=19$ (14), $\left|m_{\max }\right|=1$ (3), and $n_{\max }=7(5)$. The necessary integrals were evaluated numerically over the radial variables and analytically over the angular ones. To this end, 34 angles were considered for $W$ as well as for He-He interactions $V_{k l}$ in the interval $[0, \pi]$, which were further expanded in Legendre polynomials. For all the operators involved, the corresponding matrix elements can be found in appendix A of [23].

\subsection{Fermionic versus Bosonic clusters}

In previous work [24] we reported Hartree results regarding the binding energies and density distributions of ${ }^{4} \mathrm{He}_{N}-\mathrm{ICl}$ bosonic clusters $(N \leqslant 30)$ as well as the simulated IR spectra at a temperature of $2 \mathrm{~K}$. In a very recent study [25] the corresponding data for ${ }^{3} \mathrm{He}_{18}-\mathrm{ICl}$ fermionic clusters in all the possible spin states, obtained through Hartree-Fock calculations, were presented. In order to simulate the spectra, the quantum chemistry calculations were performed at three elongations of the ICl bond, $r=2.27,2.321$, and $2.386 \AA$. We found a logarithmic dependence $[24,25]$ of the $E_{\Lambda S}$ eigenvalues (4), on $r, E \simeq A \log (\tilde{r}-2)+B, A$ and $B$ being parameters depending on the size and nature of the cluster, and $\tilde{r}=r / \AA$. The results for ${ }^{3} \mathrm{He}_{18}-\mathrm{ICl}(\mathrm{X})$ clusters in the different spin states are summarized in table 1 .

As can be seen in the second column, the 'electronic' states correspond to either ' $\Sigma$ ' $(\Lambda=0)$ states or ' $\Pi$ ' ones $(\Lambda=1)$. As can be noted by comparing the $3 \mathrm{rd}$ columns in table 1 and table $V$ from [24] the averaged values of $\mathbf{L}^{2}$ are about 2-3 times larger in the fermionic case than in the bosonic one. As in bosonic clusters, the binding energies
Table 1. Summary of Hartree-Fock results on $\left({ }^{3} \mathrm{He}\right)_{18}-\mathrm{ICl}(X)$ clusters in all the possible spin states $(S): \Lambda$ values, averaged values of $\mathbf{L}^{2}, A$ and $B$ parameters fitting the $r$-dependence of the energy (see text), and associated VP half-widths.

\begin{tabular}{cccccc}
\hline$S$ & $\Lambda$ & $\left\langle\mathbf{L}^{2}\right\rangle(\mathrm{au})$ & $\mathrm{A}\left(\mathrm{cm}^{-1}\right)$ & $\mathrm{B}\left(\mathrm{cm}^{-1}\right)$ & $\Gamma / 2\left(\times 10^{-3} \mathrm{~cm}^{-1}\right)$ \\
\hline 0 & 0 & 158.69 & 10.01 & -192.46 & 7.25 \\
1 & 1 & 161.97 & 9.74 & -192.73 & 6.93 \\
2 & 1 & 162.18 & 9.63 & -193.82 & 6.60 \\
3 & 0 & 160.56 & 10.50 & -192.34 & 6.34 \\
4 & 0 & 175.59 & 11.27 & -191.05 & 5.36 \\
5 & 0 & 178.41 & 10.24 & -190.65 & 5.25 \\
6 & 0 & 211.71 & 7.78 & -191.73 & 6.89 \\
7 & 1 & 217.72 & 6.19 & -190.19 & 6.55 \\
8 & 0 & 222.12 & 4.67 & -188.96 & 6.47 \\
9 & 1 & 229.79 & 2.98 & -186.58 & 6.46 \\
\hline
\end{tabular}

Table 2. Summary of Hartree-Fock results on $\left({ }^{3} \mathrm{He}\right)_{N}-\mathrm{ICl}(\mathrm{X})$ fermionic clusters, $2 \leqslant N \leqslant 30$, in singlet spin states with the ICl bond length kept at equilibrium: $\Lambda$ values, binding energies $(E)$ and associated VP half-widths.

\begin{tabular}{rrrcc}
\hline$N$ & $\Lambda$ & $\left\langle\mathbf{L}^{2}\right\rangle(\mathrm{au})$ & $E\left(\mathrm{~cm}^{-1}\right)$ & $\Gamma / 2\left(\times 10^{-3} \mathrm{~cm}^{-1}\right)$ \\
\hline 2 & 0 & 19.66 & -27.25 & 0.29 \\
4 & 0 & 35.74 & -50.94 & 2.08 \\
6 & 2 & 49.39 & -75.38 & 3.04 \\
8 & 0 & 60.70 & -99.24 & 3.58 \\
10 & 0 & 72.64 & -120.77 & 5.35 \\
12 & 4 & 91.18 & -143.04 & 5.54 \\
14 & 0 & 108.52 & -164.61 & 5.82 \\
16 & 2 & 140.46 & -184.12 & 6.50 \\
18 & 0 & 158.70 & -203.28 & 7.25 \\
20 & 0 & 180.72 & -221.09 & 7.35 \\
22 & 2 & 205.34 & -238.63 & 7.57 \\
24 & 0 & 222.18 & -256.41 & 7.69 \\
26 & 4 & 264.16 & -272.69 & 7.75 \\
28 & 0 & 274.19 & -289.13 & 7.84 \\
30 & 0 & 364.13 & -305.94 & 11.88 \\
\hline
\end{tabular}

for fermions present a logarithmic dependence on the $\mathrm{ICl}$ bond length in the neighbourhood of its equilibrium value $(r=2.321 \AA)$. Note in table 1 that both the slope parameter ( $A, 4$ th column) and the binding energy at $r=3.0 \AA(B, 5$ th column) have very similar values for all the different spin states reflecting their high degree of degeneracy, a feature invariably found in fermionic clusters. As for the VP halfwidths, 6th column, small variations depending on the spin state are found.

In this work, in order to make a better contact to the experiments performed on linear dopants as OCS in nanodroplets [10, 11], (and to stress the difference in the IR spectra depending on the bosonic or fermionic nature of the solvent) we consider lower temperatures and try to extrapolate previous results to larger cluster sizes. To this end, we have performed additional Hartree-Fock calculations for the singlet state $(S=0)$ of ${ }^{3} \mathrm{He}_{N}-\mathrm{ICl}$ clusters $(2 \leqslant N \leqslant 30)$ with the ICl bond length kept fixed at its equilibrium value. The results are collected in table 2 . In spite of most of the states being of ' $\Sigma$ ' type, some ' $\Delta$ ' $(N=6,16$ and 22) and even ' $\Gamma$ ' $(N=12$ and 26$)$ states do appear. The averaged values of the squared orbital angular momentum increase almost exponentially with the cluster size $N$, being about 2-3 times larger than those corresponding to bosonic clusters (compare again with table $\mathrm{V}$ from [24]). According to (5) 
Spectral simulations of polar diatomic molecules

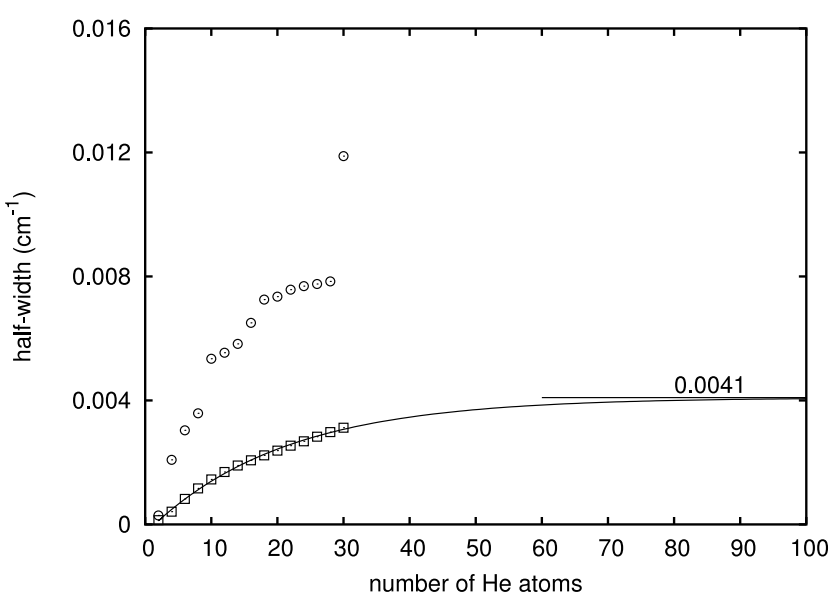

Figure 1. VP half-width of fermionic (circles) and bosonic (squares) clusters as a function of the number of solvent atoms. In the later cases, a simple extrapolation to large cluster sizes is also shown.

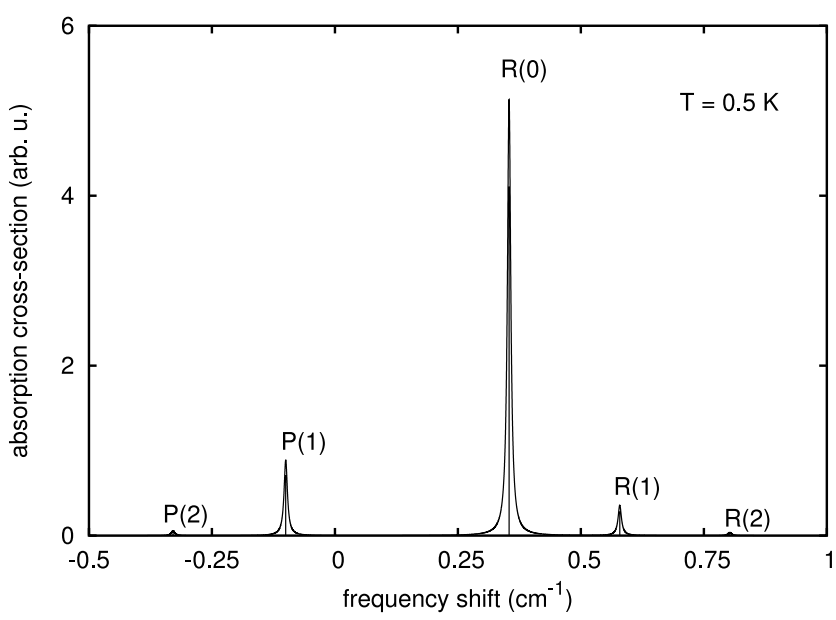

Figure 2. Simulated IR spectrum of $\operatorname{ICl}(X)$ in a nanodroplet of bosonic ${ }^{4} \mathrm{He}$ atoms at a temperature of $0.5 \mathrm{~K}$. It has been obtained by dressing the stick spectrum corresponding to a cluster of 30 bosonic ${ }^{4} \mathrm{He}$ atoms with Lorentzians of half-width equal to the extrapolated value (i.e. $\Gamma / 2=0.0041 \mathrm{~cm}^{-1}$ ).

and (6), this result implies a greater distortion of the diatomic molecule in fermionic than in bosonic environments. Unlike the quasi-linear dependence of the binding energies on $N$, the VP half-widths do not show any simple behaviour as $N$ grows in size. As illustrated in figure 1, where the VP half-widths from table 2 and table V [24] are displayed as a function of the number of solvent atoms, this is in clear contrast with the bosonic case. Thus, for bosonic clusters, this magnitude smoothly increases with cluster size and can be readily fitted to an expression of the type $\Gamma / 2(N) \sim \mathcal{G}-b \exp (-c N)$, with $\mathcal{G}=0.0041 \mathrm{~cm}^{-1}$ being the asymptotic value reached for infinite droplets. For fermionic clusters, although it clearly increases with cluster size, it shows a discontinuous behaviour and abrupt jumps at $N \approx 10,14-18$, and even more markedly at $N=30$.

As for finite clusters [24], the IR simulation in droplets is performed by considering an excitation that promotes the $\mathrm{ICl}$ dopant from the $v=0$ ground vibrational state to the $v=1$. At a very low temperature of $0.5 \mathrm{~K}$, figure 2 displays the IR absorption cross-section of $\operatorname{ICl}(X)$ solvated

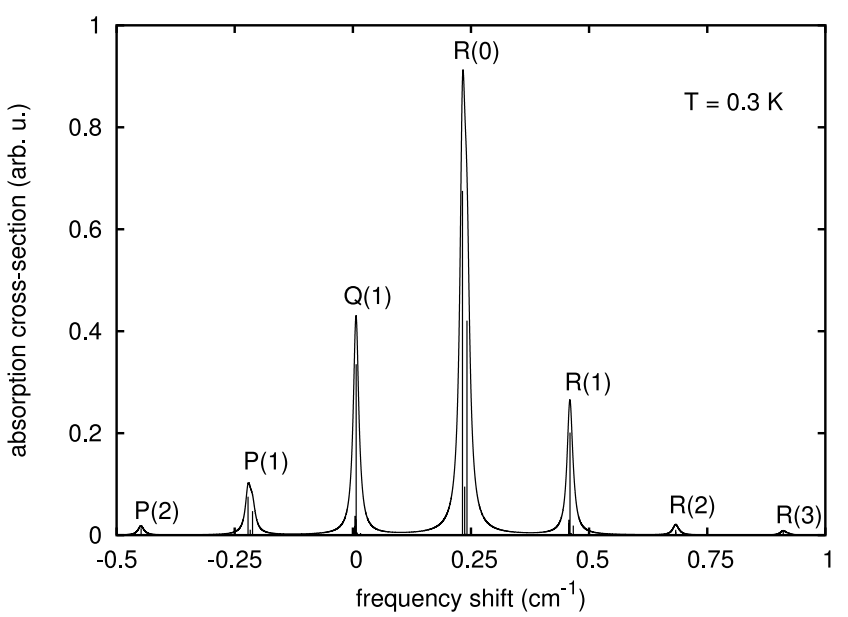

Figure 3. At $T=0.3 \mathrm{~K}$, simulated IR spectrum of the ${ }^{3} \mathrm{He}_{18}-\mathrm{ICl}(X)$ fermionic cluster showing the presence of Q-branches.

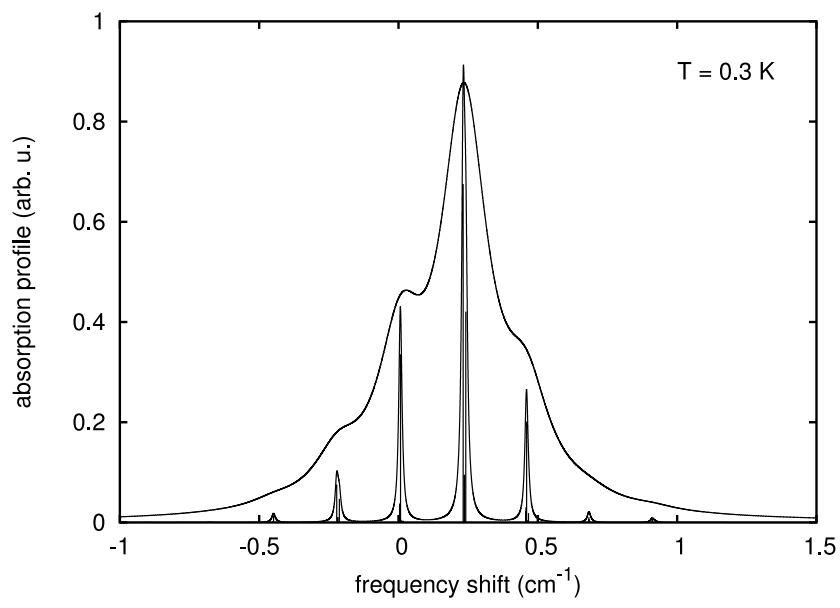

Figure 4. At $T=0.3 \mathrm{~K}$, conjectured IR spectrum of $\operatorname{ICl}(X)$ in a nanodroplet of fermionic ${ }^{3} \mathrm{He}$ atoms. It has been obtained as the spectrum of figure 3, also shown, but using Lorentzian functions 15 times wider than the original ones (see table 1).

by a very large number of bosonic ${ }^{4} \mathrm{He}$ atoms as a function of the incident photon energy (relative to the $J=0$, $v=1 \leftarrow 0,0$ forbidden transition of the bare ICl molecule, i.e. $\hbar \omega_{01}=380.084399 \mathrm{~cm}^{-1}$ ). The simulation besides an expected blueshift [24], can be considered reliable and is accomplished by dressing the lines of the stick spectrum from a cluster of $N=30$ size with Lorentzian functions of width $2 \times \mathcal{G}$. According to this model, since just ' $\Sigma$ ' states are involved for this bosonic environment, the $\mathrm{Q}$ branches are absent and only well-defined $\mathrm{P}$ and $\mathrm{R}$ ones appear. This is in good agreement with the experimental results on OCS in nanodroplets (see the upper panel of figure 1 from [10]).

In turn, and for the $\left({ }^{3} \mathrm{He}\right)_{18}-\mathrm{ICl}$ fermionic cluster, the simulated IR spectrum displayed at figure 3 shows marked differences: the $\mathrm{R}(0)$ branch remains dominant, but $\mathrm{Q}$ branches do appear as second in importance (with dominance of the transition at $J=1$ ), and all the branches come from several transitions which are a consequence of the quasidegeneracy in energy of the different spin states. Note also the larger widths of the different branches when compared with those corresponding to the bosonic scenario: for much 
larger fermionic clusters, one can expect the presence of many contributing lines leading to a complete congestion of the spectrum. So, it should look like a broad and unstructured profile showing some maximum in the neighborhood of the $\mathrm{R}(0)$ branch, as it occurs if one envisages the curve enveloping the different branches in this figure. As mentioned above, it is not possible at this stage to do a proper extrapolation of VP widths for fermionic droplets. In spite of this, a sort of enveloping of this spectrum can be easily obtained by considering much larger VP widths. So, together with the previous spectrum, figure 4 shows a conjectured IR spectrum of ICl inside fermionic droplets. It was simulated by considering VP widths 15 times larger than the original ones listed at table 1 . There is a remarkable similarity with the experiment reported on OCS, as seen in the lower panel of figure 1 from [10].

\section{Summary and outlook}

A quantum chemistry-type model to deal with clusters of a polar molecule, as $\operatorname{ICl}(X)$, solvated by $\mathrm{He}$ (boson and fermion) atoms has been outlined in this work. Both scenarios are treated at the same level of accuracy, i.e. through Hartree and Hartree-Fock calculations, respectively. The molecular interactions are represented by the sum of $a b$ initio $\mathrm{He}-\mathrm{ICl}$ triatomic surfaces plus the $\mathrm{He}-\mathrm{He}$ potential. Using an adiabatic description for the motion of the intramolecular bond distance, and assuming a simple model of VP to introduce broadening effects, IR spectra for ICl inside bosonic (fermionic) helium droplets have been simulated (conjectured). Because of the remarkable qualitative agreement reached with the experiments performed on a closely related molecule (linear OCS), we can assign a high degree of confidence to the approximations involved in the present treatment.

From the studies performed by us thus far, it seems clear that the microscopic explanation of the congested spectra for molecules immersed in fermionic clusters originates from the existence of a manifold of quasi-degenerate spin states which is further enhanced by additional allowed transitions. In this regard, it is important to underline how FCI results [30] have confirmed the high degree of degeneracy of the spin states. In the bosonic case, the ground state corresponds to the situation when all the atoms of the solvent occupy the same energy level (similarly to a Bose condensate) giving rise to a single spectral line.

The main thrust of our planned work is the development of a hierarchy of high-level $a b$ initio methods for the calculation of accurate energies and wavefunctions of doped bosonic, fermionic and mixed He clusters. Recently, we have developed an efficient FCI methodology for treating small doped fermionic clusters [30]. For larger clusters, we intend to explore computationally less expensive methods such as coupled-clusters, which have been successfully applied in nuclear physics [41-43] as well as multiconfigurational selfconsistent-field (MCSCF), and multireference-CI (MRCI) treatments, which are efficiently implemented in standard quantum chemistry packages, e.g. Molpro [44].

\section{Acknowledgments}

This work has been partially supported by the DGICYT Spanish grant FIS2004-02461 and the European project R113-CT-2003-506079. MP deL-C and RP acknowledge the support of the Spanish 'Ramón y Cajal' Programme, grant nos PDRyC-2003-001015 and PDRyC-2006-001017. JJ was supported by the Office of Basic Energy Sciences, Division of Chemical Sciences, Geosciences, and Biosciences, U S Department of Energy under Contract no DE-AC-0206CH11357. FAG acknowledges the support of the COBOL EU Network no 202-00290. The calculations presented here were performed at the Instituto de Matemáticas y Fásica Fundamental (CSIC), at CINECA (the SuperComputer Centre of the University of Bologna), and Mare-Nostrum (BSC, the Spanish National SuperComputer Centre).

\section{References}

[1] Goyal S, Schutt D L and Scoles G 1992 Phys. Rev. Lett. 69933

[2] Hartmann M, Miller R E, Toennies J and Vilesov A F 1995 Phys. Rev. Lett. 751566

[3] Callegari C, Lehmann K K, Schmied R and Scoles G 2001 J. Chem. Phys. 11510090

[4] Toennies J P and Vilesov A F 2004 Angew. Chem. Int. Edn. Engl. 432622

[5] Nauta K and Miller R E 2001 J. Chem. Phys. 11510138

[6] Nauta K and Miller R E 2002 J. Chem. Phys. 1174846

[7] Lindsay C M and Miller R E 2005 J. Chem. Phys. 122104306

[8] Tang J and McKellar A R W 2003 J. Chem. Phys. 119754

[9] McKellar A R W 2004 J. Chem. Phys. 1216868

[10] Grebenev S, Toennies J P and Vilesov A F 1998 Science 2792083

[11] Grebenev S, Hartmann M, Havenith M, Sartakov B, Toennies J P and Vilesov A F 2000 J. Chem. Phys. 1124485

[12] Baroni S and Moroni S 1999 in Quantum Monte Carlo Methods in Physics and Chemistry vol 525, ed P Nightingale and C J Umrigar, NATO Series Mathematical and Physical Sciences (Boston: Kluwer)

[13] Zillich Z E, Paesani F, Kwon Y and Whaley K B 2005 J. Chem. Phys. 123114301

[14] Barranco M, Guardiola R, Hernández S, Mayol R, Navarro J and Pí M 2006 J. Low. Temp. Phys. 1421

[15] Anderson J D 1975 J. Chem. Phys. 631499

[16] Sola E, Casulleras J and Boronat J 2006 Phys. Rev. B 73 092515

[17] Jungwirth P and Krylov A 2001 J. Chem. Phys. 11510214

[18] Heidenreich A, Even U and Jortner J 2001 J. Chem. Phys. 115 10175

[19] Heidenreich A and Jortner J 2003 J. Chem. Phys. 11810101

[20] Felker P M 2006 J. Chem. Phys. 125184313

[21] López-Durán D, de Lara-Castells M P, Delgado-Barrio G, Villarreal P, Paola C D, Gianturco F A and Jellinek J 2004 Phys. Rev. Lett. 93053401

[22] López-Durán D, de Lara-Castells M P, Delgado-Barrio G, Villarreal P, Paola C D, Gianturco F A and Jellinek J 2004 J. Chem. Phys. 1212975

[23] de Lara-Castells M P, López-Durán D, Delgado-Barrio G, Villarreal P, Paola C D, Gianturco F A and Jellinek J 2005 Phys. Rev. A 71033203

[24] de Lara-Castells M P, Prosmiti R, Delgado-Barrio G, López-Durán D, Villarreal P, Gianturco F A and Jellinek J 2006 Phys. Rev. A 74053201

[25] de Lara-Castells M P, Prosmiti R, López-Durán D, Delgado-Barrio G, Villarreal P, Gianturco F A and Jellinek J 2007 Int. J. Quantum. Chem. at press 
[26] Valdés A, Prosmiti R, Villarreal P and Delgado-Barrio G 2006 J. Chem. Phys. 125014313

[27] Prosmiti R, Cunha C, Villarreal P and Delgado-Barrio G 2002 J. Chem. Phys. 1177017

[28] Villarreal P, Roncero O and Delgado-Barrio G 1994 J. Chem. Phys. 1012217

[29] Roncero O, Pérez-de Tudela R, de Lara-Castells M P, Prosmiti R, Delgado-Barrio G and Villarreal P 2007 Int. J. Quantum. Chem. $\mathbf{1 0 7}$ at press

[30] de Lara-Castells M P, Delgado-Barrio G, Villarreal P and Mitrushchenkov A O 2006 J. Chem. Phys. 125221101

[31] Barletta P, Fabrocini A, Kievsky A, Navarro J and Polls A 2003 Phys. Rev. A 68053205

[32] Fernández-Rico J 1990 Self-consistent Field: Theory and Applications (Studies in Physical and Theoretical Chemistry vol 70) ed Carbo R and Klobukowski M (Amsterdam: Elsevier)

[33] Fernández-Rico J, de la Vega J M G, Paniagua M and Fernández-Alonso J I 1983 J. Chem. Phys. 794407

[34] Miguel B, Bastida A, Zúñiga J, Requena A and Halberstadt N 2001 Faraday Discuss. 118257

[35] Hernández M I, González-Lezana T, Buchachenko A, Prosmiti R, de Lara-Castells M P, Delgado-Barrio G and
Villarreal P 2003 Recent Research Developments in Chemical Physics vol 4 (Kerala, India: Transworld Research Network) p 1

[36] Villarreal P, Varadé A and Delgado-Barrio G 1989 J. Chem. Phys. 902684

[37] Hernández M I, Garcáa-Vela A, Campos-Martánez J, Roncero O, Villarreal P and Delgado-Barrio G 2002 Comput. Phys. Commun. 14597

[38] Beswick J A and Delgado-Barrio G 1980 J. Chem. Phys. 73 3653

[39] Huber K P and Herzberg G 1979 Constants of Diatomic Molecules (New York: Van Nostrand Reinhold)

[40] Aziz R A and Slaman M J 1991 J. Chem. Phys. 948047

[41] Kowalski K, Dean D J, Hjorth-Jensen M, Papenbrock T and Piecuch P 2004 Phys. Rev. Lett. 92132001

[42] Wloch M, Dean D J, Gour J R, Hjorth-Jensen M, Kowalski K, Papenbrock T and Piecuch P 2005 Phys. Rev. Lett. 94 212501

[43] Gour J R, Piecuch P, Hjorth-Jensen M, Wloch M and Dean D J 2006 Phys. Rev. C 74024310

[44] Werner H-J et al 2006 Molpro, Version 2006.1, A Package of ab initio Programs see http://www.molpro.net, (Cardiff, UK) 